\title{
Constructing a Knowledge Base for Gene Regulatory Dynamics by Formal Concept Analysis Methods
}

\author{
Johannes Wollbold ${ }^{12}$, Reinhard Guthke ${ }^{2}$, and Bernhard Ganter ${ }^{1}$ \\ 1 University of Technology, Institute of Algebra, Dresden, Germany \\ http://www .math.tu-dresden.de/alg/algebra.html \\ jwollbold@gmx.de \\ 2 Leibniz Institute for Natural Product Research and Infection Biology - \\ Hans-Knöll-Institute (HKI) Jena, Germany
}

\begin{abstract}
Our aim is to build a set of rules, such that reasoning over temporal dependencies within gene regulatory networks is possible. The underlying transitions may be obtained by discretizing observed time series, or they are generated based on existing knowledge, e.g. by Boolean networks or their nondeterministic generalization. We use the mathematical discipline of formal concept analysis (FCA), which has been applied successfully in domains as knowledge representation, data mining or software engineering. By the attribute exploration algorithm, an expert or a supporting computer program is enabled to decide about the validity of a minimal set of implications and thus to construct a sound and complete knowledge base. From this all valid implications are derivable that relate to the selected properties of a set of genes. We present results of our method for the initiation of sporulation in Bacillus subtilis. However the formal structures are exhibited in a most general manner. Therefore the approach may be adapted to signal transduction or metabolic networks, as well as to discrete temporal transitions in many biological and nonbiological areas.
\end{abstract}

Keywords: complete lattices, reasoning, temporal logic, gene expression

\section{Introduction}

As the mathematical methodology of formal concept analysis (FCA) is little known within systems biology, we give a short overview of its history and purposes. During the early years 1980, FCA emerged within the community of set and order theorists, algebraists and discrete mathematicians. Its first aim was to find a new, concrete and meaningful approach to the understanding of complete lattices (ordered sets such that for every subset the supremum and the infimum exist). The following discovery showed to be very fruitful: Every complete lattice is representable as a hierarchy of concepts, which were conceived as sets of objects sharing a maximal set of attributes. This paved the way for using the developed field of lattice theory for a transparent and complete representation of very different types of knowledge. FCA was inspired by the pedagogue Hartmut 
von Hentig [7] and his program of restructuring sciences, with a view to interdisciplinary collaboration and democratic control. The philosophical background goes back to Charles S. Peirce (1839 - 1914), who condensed some of his main ideas to the pragmatic maxim:

Consider what effects, that might conceivably have practical bearings, we conceive the objects of our conception to have. Then, our conception of these effects is the whole of our conception of the object. $[14,5.402]$

In that tradition, FCA aims at unfolding the observable, elementary properties defining the objects subsumed by scientific concepts. If applied to temporal transitions, effects of homogeneous classes of states can be modeled and predicted in a clear and concise manner. Thus FCA seems to be appropriate to describe causality - and the limits of its understanding.

At present, FCA is a richly developed mathematical theory, and there are practical applications in various fields as data and text mining, knowledge management, semantic web, software engineering or economics [3]. FCA has been used for the analysis of gene expression data in [2] and [13], but this is the first approach of applying it to model (gene) regulatory networks. The mathematical framework of FCA is very general and open, such that multifarious refinements are possible, according to current approaches of modeling dynamics within systems biology. On the other hand, we developed a formal structure for general discrete temporal transitions. They occur in a variety of domains: control of engineering processes, development of the values of variables or objects in a computer program, change of interactions in social networks, a piece of music, etc.

In this paper, however, the examples are uniquely biological. The purpose is to construct a knowledge base for reasoning about temporal dependencies within gene regulatory or signal transduction networks, by the attribute exploration algorithm: For a given set of interesting properties, it builds a sound, complete and nonredundant knowledge base. This minimal set of rules has to be checked by an expert or a computer program, e.g. by comparison of knowledge based predictions with data.

Since there exist relatively fixed thresholds of activation for many genes, it is a common abstraction to consider only two expression levels off and on. The classical approach of Boolean networks [8] is able to capture essential dynamic aspects of regulatory networks. Our present work is based on it, which also makes it possible to use mathematical and logical derivations for deciding many rules automatically and for a scaling up to larger networks. Nevertheless, the introduction of more fine-grained expression levels remains possible, e.g. in the sense of qualitative reasoning [11]. Further, this work is influenced by computation tree logic [1], automata theory and a FCA modeling of temporal transitions in [15]. Temporal concept analysis as developed by K.E. Wolff [3, p. 127-148] is more directed toward a structured visualization of experimental time series then toward temporal logic. We applied it to the analysis of gene expression data in $[19]$. 
In Section 2, the general mathematical framework will be developed. Section 3 gives results for a $B$. subtilis Boolean network. In Section 4, we will discuss the potential of the method and make some proposals for improving it by solving mathematical problems which have emerged from the applications.

\section{Methods}

\subsection{Fundamental Structures of Formal Concept Analysis}

One of the classical aims of FCA is the structured, compact but complete visualization of a data set by a conceptual hierarchy. We briefly introduce its basic definitions; for an easy example see http://www.upriss.org.uk/fca/fca.html.

Definition 1. A formal context $(G, M, I)$ defines a relation $I \subseteq G \times M$ between objects from a set $G$ and attributes from a set $M$. The set of the attributes common to all objects in $A \subseteq G$ is denoted by the '-operator:

$$
A^{\prime}:=\{m \in M \mid g I m \text { for all } g \in A\} .
$$

The set of the objects sharing all attributes in $B \subseteq M$ is

$$
B^{\prime}:=\{g \in G \mid g I m \text { for all } m \in B\} .
$$

Definition 2. A formal concept of the context $(G, M, I)$ is a pair $(A, B)$ with $A \subseteq G, B \subseteq M, A^{\prime}=B$ and $B^{\prime}=A$. $A$ is the extent, $B$ the intent of the concept $(A, B)$.

Thus a formal context $(G, M, I)$ is a special, but universally applicable type of a data table, provided with two operators $\mathcal{C}_{M}: \mathcal{P}(M) \rightarrow \mathcal{P}(M), B \subseteq M \mapsto$ $B^{\prime \prime}$ and $\mathcal{C}_{G}: \mathcal{P}(G) \rightarrow \mathcal{P}(G), A \subset G \mapsto A^{\prime \prime}$. It is easy to see that they are closure operators, with the properties monotony, extension and idempotency [4, Definition 14]. It follows that the set of all extents resp. intents of a formal context is a closure system, i.e. it is closed under intersections [4, Theorem 1].

Formal concepts can be ordered by set inclusion of the extents or - dually, with the inverse order relation - of the intents. With this order, the set of all concepts of a given formal context is a complete lattice [4, Theorem 3] (Figure 1).

During the interactive attribute exploration algorithm [4, p. 85ff.], an expert is asked about the general validity of basic implications $A \rightarrow B$ between the attributes of a given formal context $(G, M, I)$. An implication has the meaning: "If an object $g \in G$ has all attributes $a \in A \subseteq M$, then it has also all attributes $b \in B \subseteq M$." If the expert denies, s/he must provide a counterexample, i.e. a new object of the context. If $\mathrm{s} /$ he accepts, the implication is added to the stem base of the - possibly enlarged - context. A theorem by Duquenne-Guiges [4, Theorem 8 ] ensures that every implication semantically valid in the underlying formal context can be derived syntactically from this minimal set by the Armstrong rules [4, Proposition 21]. In many applications, one is merely interested in the implicational logic of a given formal context, and there is no need for an expert to confirm the implications. 


\subsection{Constructing the Knowledge Base - Summary of the Method}

We start with two sets:

- The universe $E$. The elements of $E$ represent the entities of the world which we are interested in

- The set $F$ (fluents) denotes changing properties of the entities.

A state $\varphi \in G$ is an assignment of values in $F$ to the variables $e \in E$, hence it is defined by a specific choice of attributes $m \in M \subseteq E \times F .{ }^{3}$ By means of a state context (Definition 3, Table 3 left part), temporal data can be translated into the language of FCA. The dynamics is modeled by a binary relation $R \subseteq G \times G$ on the set of states, which gives rise to a transition context $\mathbb{K}$ (Definition 4, Table 1): the objects are transitions (elements of the relation) and the attributes the values of the entities defining the input and output state of a transition. This data table may reflect observations repeated at different time points, or the transitions may be generated by a dynamic model. As to the latter, we are focusing here on Boolean networks, i.e sets of Boolean functions for each entity (Definition 5).

It is promising to consider the transitive closure of $R$. Objects of the transitive context $\mathbb{K}_{t}$ (Definition 6, Table 2) then are pairs of states such that the output state emerges from the input state by some transition sequence of arbitrary length. Finally we extend the state context $\mathbb{K}_{s}$ by the temporal attributes always $(m)$, eventually $(m)$ and never $(m)$, which are determined by the given transitions (Definition 3, Table 3).

The defined mathematical structures may be used in various ways. For instance, one could evaluate - i.e. generalize implications or reject them supposing outliers or by reason of special conditions - experimental time series by comparison with existing knowledge. Our general procedure is the following:

1. Discretize a set of time series of gene expression measurements and transform it to an observed transition context $\mathbb{K}^{\text {obs }}$.

2. For a set of interesting genes, translate interactions from biological literature and databases into a Boolean network.

3. Construct the transition context $\mathbb{K}$ by a simulation starting from a set of states, e.g. the initial states of $\mathbb{K}^{o b s}$ or all states (for small networks).

4. Derive the respective transitive contexts $\mathbb{K}_{t}$ and $\mathbb{K}_{t}^{o b s}$.

5. Perform attribute exploration of $\mathbb{K}_{t}$. Decide about an implication $A \rightarrow B$, $A, B \subseteq M$, by checking its validity in $\mathbb{K}_{t}^{o b s}$ and/or by searching for supplementary knowledge. Possibly provide a counterexample from $\mathbb{K}_{t}^{\text {obs }}$.

6. Answer queries from the modified context $\mathbb{K}_{t}$ and from its stem base.

In step 5., automatic decision criteria could be thresholds of support $q=\mid(A \cup$ $B)^{\prime} \mid$ and confidence $p=\frac{\left|(A \cup B)^{\prime}\right|}{\left|A^{\prime}\right|}$ for an implication in $\mathbb{K}_{t}^{\text {obs }}$. A weak criterion

\footnotetext{
3 Thus - as usual - states with the same variable values are identified. It would also be possible to distinguish them as situations by introducing a new attribute, e.g. "time interval".
} 
is to reject only implications with support 0 (but if no object in $\mathbb{K}_{t}^{o b s}$ has all attributes from $\mathrm{A}$, the implication is not violated). In [18], a strong criterion has been applied: implications of $\mathbb{K}_{t}$ had to be valid also in the observed context $(p=1)$. This is equivalent to an exploration of the union of the two contexts.

In Section 3 we will analyse pure knowledge based simulations; the validation by data and experimental literature had been done before in [5]. For that reason, in step 5 . the stem base is computed automatically, without further confirmation by an expert.

Step 2. could be supported by text mining software. Then attribute exploration provides strong criteria of validation. We implemented the steps 1., 3 . and 4. in R [www.r-project.org]. For step 5., we used the Java tool Concept Explorer [http://sourceforge.net/projects/conexp]. The output was translated with $\mathrm{R}$ into a PROLOG knowledge base. The $\mathrm{R}$ scripts are available on request.

\subsection{Definition of the Relevant Formal Contexts}

With given sets $E$ and $F$, we define a state as a map $\varphi: E \rightarrow F$. To explore static features of states, the following formal context ${ }^{4}$ is defined.

Definition 3. Given two sets $E$ (entities) and $F$ (fluents), a state context is a formal context $(G, M, I)$ with $G \subseteq F^{E}:=\{\varphi: E \rightarrow F\}$ and $M \subseteq E \times F$; its relation $I$ is given as $\varphi I(e, f) \Leftrightarrow \varphi(e)=f$, for all $\varphi \in G, e \in E$ and $f \in F$.

Definition 4. Given a state context $(G, M, I)$ and a relation $R \subseteq G \times G$, a transition context $\mathbb{K}$ is the context $(R, M \times\{i n$, out $\}, \tilde{I})$ with the property

$$
\forall i \in\{\text { in }, \text { out }\}:\left(\varphi^{\text {in }}, \varphi^{\text {out }}\right) \tilde{I}(e, f, i) \Leftrightarrow \varphi^{i}(e)=f .
$$

Transitions may be generated by a Boolean network:

Definition 5. Let $E$ be an arbritray set of entities, $F:=\{0,1\}$ (fluents), and states $G \subseteq F^{E}$. Then a transition function $F^{E} \rightarrow F^{E}$ is called a Boolean network.

We will identify the elements of $F$ with,-+ or off, on respectively. This definition is subsumed by the definition of a dynamic network in [12, Definition p. 34], with a set of variables $E$ and state sets $X_{1}=\ldots=X_{n}=F$. We use a parallel update schedule, i.e. the order relation on $E$ is empty. Boolean networks may be generalized in order to include nondeterminism; then different output states $\varphi^{\text {out }}$ are generated from a single input state $\varphi^{\text {in }}$ (see Section 3, compare [18]).

Definition 6. A transition context $\mathbb{K}$ with a transitively closed relation $t(R) \subseteq$ $G \times G$ is called a transitive context $\mathbb{K}_{t}$.

\footnotetext{
${ }^{4}$ It is equivalent to a "many-valued context" with "nominal scale" [4, Section 1.3], $[18$, p. 123]. For better readability, we draw the contexts in the latter form (Table 3). Deriving a one-valued context according to Definition 3 is obvious: each many-valued attribute $e$ is replaced by $\{(e, f) \mid f \in F\})$, e.g. SigA by SigA.off and SigA.on. If an attribute $e$ takes exactly one of these values, negation of on and off is expressed. Other kinds of scaling like the interordinal scale could be interesting, if there are more than two levels $(|F|>2)$.
} 
Table 1. A transition context for the states of Table 3, with all attributes that are changing during the small simulation, as well as Spo0A and Spo0AP.

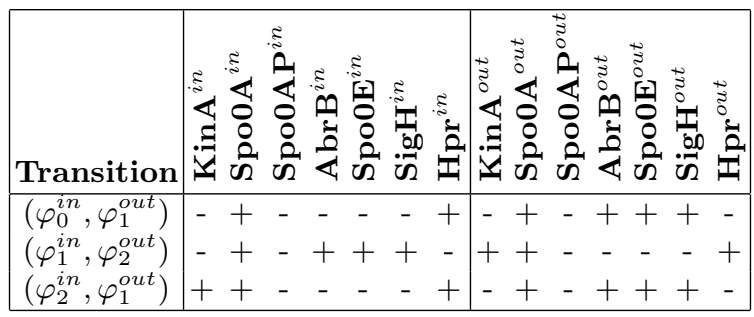

Table 2. The transitive context derived from the transition context of Table 1 .

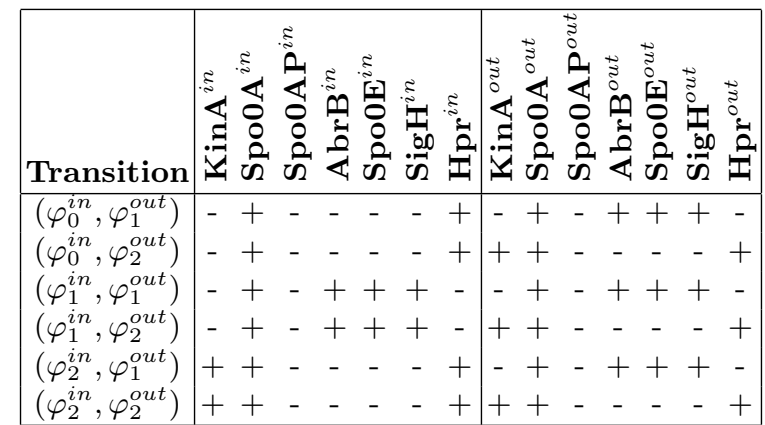

Definition 7. A state context $\mathbb{K}=(G, M, I)$ is extended to a formal context $\mathbb{K}_{s}=\left(G, M \cup T, I \cup I_{T}\right)$ by a set of temporal attributes $T:=\{$ always $(m) \mid m \in$ $M\} \cup\{$ never $(m) \mid m \in M\} \cup\{$ eventually $(m) \mid m \in M\}$. Let $\varphi^{i n} \in G, m=$ $(e, f), e \in E, f \in F$, and $t(R) \subseteq G \times G$ a transitively closed relation. The relation $I_{T}$ of $\mathbb{K}_{s}$ then is defined as follows:

$$
\begin{aligned}
\varphi^{\text {in }} I_{T} \text { always }(m) & \Leftrightarrow \forall\left(\varphi^{\text {in }}, \varphi^{\text {out }}\right) \in t(R): \varphi^{\text {out }}(e)=f \\
\varphi^{\text {in }} I_{T} \text { never }(m) & \Leftrightarrow \forall\left(\varphi^{\text {in }}, \varphi^{\text {out }}\right) \in t(R): \varphi^{\text {out }}(e) \neq f \\
\varphi^{\text {in }} I_{T} \text { eventually }(m) & \Leftrightarrow \exists\left(\varphi^{\text {in }}, \varphi^{\text {out }}\right) \in t(R): \varphi^{\text {out }}(e)=f
\end{aligned}
$$

For $B \subseteq T$, set always $(B):=\left\{\left\{\right.\right.$ always $\left(b_{1}\right), \ldots$, always $\left.\left.\left(b_{i}\right)\right\} \mid b_{1}, \ldots, b_{i} \in B\right\}$, and analogously never $(B)$ and eventually $(B)$.

The attributes will be abbreviated to $\operatorname{alw}(m), \operatorname{nev}(m)$ and ev $(m)$. In a nondeterministic setting, alw $(m)$ and nev $(m)$ refer to all possible transition paths starting from $\varphi^{i n}, \operatorname{ev}(m)$ to the existence of a path.

\subsection{Dependency of Contexts and Background Knowledge}

In the following we will present first mathematical results that can improve computability; they are not necessary for the understanding of the application in Section 3. By entering background knowledge (not necessarily implications) 
Table 3. Left part: A state context corresponding to a simulation starting from a $B$. subtilis state without nutritional stress (see Section 3.1, [16, Table 4]). +: on, -: off. Right part: extension by temporal attributes. Here they are the same for all states, since these reach the attractor (limit state cycle) $\left\{\varphi_{1}, \varphi_{2}\right\}$ after at most one time step.

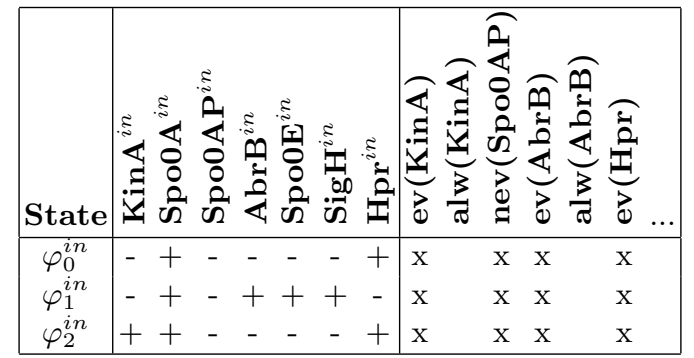

prior to an attribute exploration, the algorithm may be shortened considerably [3, p. 101-113]. We searched for first order logic background formula in order to use the results of an attribute exploration for the exploration of the next context in the hierarchy. Then the implications of the latter context are derivable from this background knowledge and a reduced set of new implications. Also during the exploration of one context, implications can be decided automatically based on already accepted implications. In this way the expert is enabled to concentrate on really interesting hypotheses. Thus, the implications of a state context hold in the input and output part of the corresponding transition context (for an example see [15, p. 149f.]). Related to transitive and extended state contexts, the subsequent result holds:

Proposition 1. Let $\mathbb{K}_{s}=\left(G, M \cup T, I \cup I_{T}\right)$ an extended state context. Suppose the relation $t(R) \subseteq G \times G$ is the object set of the transitive context $\mathbb{K}_{t}=$ $(t(R), M \times\{$ in, out $\}, \tilde{I})$. Then the following entailments between implications of both contexts are valid:

$$
\begin{aligned}
B^{\text {in }} & \rightarrow m^{\text {out }} \text { in } \mathbb{K}_{t} \equiv B \rightarrow \operatorname{always}(m) \text { in } \mathbb{K}_{s} \\
B^{\text {in }} & \rightarrow m^{\text {out }} \text { in } \mathbb{K}_{t} \models B \rightarrow \operatorname{eventually}(m) \text { in } \mathbb{K}_{s} \\
B^{\text {out }} & \rightarrow m^{\text {out }} \text { in } \mathbb{K}_{t} \models \operatorname{always}(B) \rightarrow \operatorname{always}(m) \text { in } \mathbb{K}_{s} \\
B^{\text {out }} & \rightarrow m^{\text {out }} \text { in } \mathbb{K}_{t} \models \operatorname{eventually}(B) \rightarrow \operatorname{eventually}(m) \text { in } \mathbb{K}_{s} \\
B^{\text {in }} \cup m^{\text {out }} \rightarrow \perp \text { in } \mathbb{K}_{t} & \models B \rightarrow \text { never }(m) \text { in } \mathbb{K}_{s}
\end{aligned}
$$

If the latter implication does not follow from the stem base of $\mathbb{K}_{t}$, this is equivalent to $B \rightarrow$ eventually $(m)$ in $\mathbb{K}_{s}$.

Proof. The proofs are straightforward from the definitions.

In order to get a complete overview on valid entailments, as a first step we performed rule exploration [20] of the following test context, i.e. exploration of Horn rules instead of implications, thus variables are admitted: The objects 
are all possible $\mathbb{K}_{t}$ respectively the corresponding $\mathbb{K}_{s}$, and the attributes are the following classes of implications with "homogeneous" premises. Then the explored rules for implications correspond to entailments valid for the semantics given by the objects, the transitive contexts. The sets are nonempty subsets of $M, m=(e, f), f \in F:=\{0,1\}$, and $m \in B_{0}, C_{0}\left(B_{1}, C_{1}\right) \Rightarrow \varphi(e)=0$ (1). We suppose that all states and transitions are completely defined.
1. $B^{i n} \rightarrow C^{i n}$
2. $B^{\text {in }} \rightarrow C_{0}^{\text {out }} \equiv B^{\text {in }} \rightarrow \operatorname{nev}\left(C_{1}\right)$
3. $B^{\text {in }} \rightarrow C_{1}^{\text {out }} \equiv B^{\text {in }} \rightarrow \operatorname{alw}\left(C_{1}\right)$
4. $B^{i n} \rightarrow \operatorname{ev}\left(C_{1}\right)$
5. $B_{0}^{\text {out }} \rightarrow C^{\text {in }}$
6. $B_{1}^{\text {out }} \rightarrow C^{\text {in }}$
7. $\operatorname{ev}\left(B_{1}\right) \rightarrow C^{i n}$
8. $\operatorname{alw}\left(B_{1}\right) \rightarrow C^{i n}$
9. $\operatorname{nev}\left(B_{1}\right) \rightarrow C^{i n}$
10. $B_{0}^{\text {out }} \rightarrow C_{0}^{\text {out }}$
11. $B_{0}^{\text {out }} \rightarrow C_{1}^{\text {out }}$
12. $B_{1}^{\text {out }} \rightarrow C_{0}^{\text {out }}$
13. $B_{1}^{\text {out }} \rightarrow C_{1}^{\text {out }}$
14. $\mathrm{ev}\left(B_{1}\right) \rightarrow \operatorname{ev}\left(C_{1}\right)$
15. $\mathrm{ev}\left(B_{1}\right) \rightarrow \operatorname{alw}\left(C_{1}\right)$
16. $\mathrm{ev}\left(B_{1}\right) \rightarrow \operatorname{nev}\left(C_{1}\right)$
17. $\operatorname{alw}\left(B_{1}\right) \rightarrow \operatorname{ev}\left(C_{1}\right)$
18. $\operatorname{alw}\left(B_{1}\right) \rightarrow \operatorname{alw}\left(C_{1}\right)$
19. $\operatorname{alw}\left(B_{1}\right) \rightarrow \operatorname{nev}\left(C_{1}\right)$
20. $\operatorname{nev}\left(B_{1}\right) \rightarrow \operatorname{ev}\left(C_{1}\right)$
21. $\operatorname{nev}\left(B_{1}\right) \rightarrow \operatorname{alw}\left(C_{1}\right)$
22. $\operatorname{nev}\left(B_{1}\right) \rightarrow \operatorname{nev}\left(C_{1}\right)$

The equivalences in 2. and 3. follow from Proposition 1(2). Since the implications comprising input attributes are independent from those related only to output attributes, rule exploration was performed (almost) independently for the first 9 and the remaining 13 implications. Results for the second part are shown here.

The exploration started from a hypothetical context as single object of the test context, where no implications were valid. Before, we had added 25 known entailments as background rules (BR), like those of Proposition 1 or following from the definitions, like alw $\left(B_{1}\right) \rightarrow \mathrm{ev}\left(B_{1}\right)$. A counterexample represents a significant class of contexts. They had to be chosen carefully, since an object not having its maximal attribute set might preclude a valid entailment. ${ }^{5}$ The exploration resulted in the following stem base of only 14 entailments. Most of them are background rules (they are accepted automatically during the exploration), but not all of these are needed in order to derive all valid entailments between the chosen implications. This demonstrates the effectivity and minimality of the algorithm. Entailments 5., 6., 7. and 10. were newly found.

1. $\operatorname{nev}\left(B_{1}\right) \rightarrow \operatorname{alw}\left(C_{1}\right) \models \operatorname{nev}\left(B_{1}\right) \rightarrow \operatorname{ev}\left(C_{1}\right)(\operatorname{BR} 1)$

2. $\operatorname{nev}\left(B_{1}\right) \rightarrow \operatorname{ev}\left(C_{1}\right), \operatorname{nev}\left(B_{1}\right) \rightarrow \operatorname{nev}\left(C_{1}\right) \models \perp(\operatorname{BR} 11)$

3. $\operatorname{alw}\left(B_{1}\right) \rightarrow \operatorname{alw}\left(C_{1}\right) \models \operatorname{alw}\left(B_{1}\right) \rightarrow \operatorname{ev}\left(C_{1}\right)(\operatorname{BR} 2)$

4. $\operatorname{alw}\left(B_{1}\right) \rightarrow \operatorname{ev}\left(C_{1}\right), \operatorname{alw}\left(B_{1}\right) \rightarrow \operatorname{nev}\left(C_{1}\right) \models \perp($ BR 14)

5. $\operatorname{ev}\left(B_{1}\right) \rightarrow \operatorname{nev}\left(C_{1}\right), \operatorname{nev}\left(B_{1}\right) \rightarrow \operatorname{nev}\left(C_{1}\right)=B^{\text {in }} \rightarrow C_{0}^{\text {out }}, B_{0}^{\text {out }} \rightarrow C_{0}^{\text {out }}, B_{1}^{\text {out }}$ $\rightarrow C_{0}^{\text {out }}$

6. $\operatorname{ev}\left(B_{1}\right) \rightarrow \operatorname{nev}\left(C_{1}\right), \operatorname{alw}\left(B_{1}\right) \rightarrow \operatorname{nev}\left(C_{1}\right) \models B_{1}^{\text {out }} \rightarrow C_{0}^{\text {out }}$

7. $\operatorname{ev}\left(B_{1}\right) \rightarrow \operatorname{nev}\left(C_{1}\right), \operatorname{alw}\left(B_{1}\right) \rightarrow \operatorname{ev}\left(C_{1}\right) \models \perp$

${ }^{5}$ Thus the attribute set of a counterexample must be a concept intent in the final test context. 
8. $\left.\left.\operatorname{ev}\left(B_{1}\right) \rightarrow \operatorname{alw}\left(C_{1}\right) \models \operatorname{ev}\left(B_{1}\right) \rightarrow \operatorname{ev}\left(C_{1}\right), \operatorname{alw}\left(B_{1}\right)\right) \rightarrow \operatorname{ev}\left(C_{1}\right), \operatorname{alw}\left(B_{1}\right)\right) \rightarrow$ $\operatorname{alw}\left(C_{1}\right)(\mathrm{BR} 3)$

9. $\operatorname{ev}\left(B_{1}\right) \rightarrow \operatorname{ev}\left(C_{1}\right) \models \operatorname{alw}\left(B_{1}\right) \rightarrow \operatorname{ev}\left(C_{1}\right)(\operatorname{BR} 4)$

10. $\mathrm{ev}\left(B_{1}\right) \rightarrow \operatorname{ev}\left(C_{1}\right), \operatorname{nev}\left(B_{1}\right) \rightarrow \operatorname{ev}\left(C_{1}\right) \models B^{\text {in }} \rightarrow C_{1}^{\text {out }}, B_{0}^{\text {out }} \rightarrow C_{1}^{\text {out }}, B_{1}^{\text {out }} \rightarrow$ $C_{1}^{\text {out }}, \operatorname{ev}\left(B_{1}\right) \rightarrow \operatorname{alw}\left(C_{1}\right)$

11. $B_{1}^{\text {out }} \rightarrow C_{1}^{\text {out }} \models \operatorname{ev}\left(B_{1}\right) \rightarrow \operatorname{ev}\left(C_{1}\right), \operatorname{alw}\left(B_{1}\right) \rightarrow \operatorname{ev}\left(C_{1}\right), \operatorname{alw}\left(B_{1}\right) \rightarrow \operatorname{alw}\left(C_{1}\right)$ (BR 4 , BR $5 \Leftarrow$ Proposition 1(4)(5))

12. $B_{1}^{\text {out }} \rightarrow C_{0}^{\text {out }} \models \operatorname{alw}\left(B_{1}\right) \rightarrow \operatorname{nev}\left(C_{1}\right)($ BR $9 \Leftarrow$ Proposition $1(4))$

13. $B_{0}^{\text {out }} \rightarrow C_{1}^{\text {out }} \models \operatorname{nev}\left(B_{1}\right) \rightarrow \operatorname{ev}\left(C_{1}\right)$, nev $\left(B_{1}\right) \rightarrow \operatorname{alw}\left(C_{1}\right)$ (BR $1,10 \Leftarrow$ Proposition 1(4))

14. $B_{0}^{\text {out }} \rightarrow C_{0}^{\text {out }} \models \operatorname{nev}\left(B_{1}\right) \rightarrow \operatorname{nev}\left(C_{1}\right)($ BR $6 \Leftarrow$ Proposition $1(4))$

It remains to prove the rules of this stem base, which is easy; we are giving some hints: BR $1,2,3$, and 4 are based on $\operatorname{alw}(A) \rightarrow \operatorname{ev}(A), A \subseteq M$, and BR 11 and 14 on $\operatorname{nev}\left(C_{1}\right), \operatorname{ev}\left(C_{1}\right) \rightarrow \perp$ ( $\perp=$ set of all attributes, and the corresponding object set is empty).

7.: Since nev $\left(C_{1}\right)$ and ev $\left(C_{1}\right)$ do not occur together by definition, the combination of the two implications has support 0 in the test context. In the underlying contexts, the premise alw $\left(B_{1}\right)$ (a subcase of ev( $\left.B_{1}\right)$ ) is no attribute of any state. The implication alw $\left(B_{1}\right) \rightarrow \perp$ holds, which has not been considered explicitly.

10.: Inversely, in all possible cases the states / transitions have the attribute ev $\left(C_{1}\right)$, and therefore also alw $\left(C_{1}\right)$ and $C_{1}^{\text {out }}$. Explicitly: $\top \rightarrow \operatorname{ev}\left(C_{1}\right), \top \rightarrow$ $\operatorname{alw}\left(C_{1}\right), \top \rightarrow C_{1}^{\text {out }} .5$. is a parallel rule concerning nev $\left(C_{1}\right)$.

Rules 7. and 10. suggest that implications with empty premise $T$ or conclusion $\perp$ should be considered explicitly. If the counterexamples have maximal attribute sets, as a conclusion it can be stated that we have derived a set of rules representing a minimal, sound and complete entailment calculus for the selected classes of implications for transition and state contexts.

\section{Results: Sporulation in Bacillus subtilis}

In order to demonstrate the characteristics of the proposed method, we will apply it to a gene regulatory network assembled in [5] and transformed to a Petri net as well as a Boolean network in [16].

B. subtilis is a gram positive soil bacterium. Under extreme environmental stress, it produces a single endospore, which can survive ultraviolet or gamma radiation, acid, hours of boiling or long periods of starvation. The bacterium leaves the vegetative growth phase in favour of a dramatically changed and highly energy consuming behaviour, and it dies at the end of the sporulation process. This corresponds to a switch between two clearly distinguished genetic programs, which are complex but comparatively well understood.

By literature and database search, de Jong et al. [5] identified 12 main regulators, constructed a model of piecewise linear differential equations and obtained realistic simulation results. An exogenous signal (starvation) triggers the phosphorylation of the transcription factor Spo0A to Spo0AP by the kinase KinA; this process is reversible by the phosphatase Spo0E. Spo0AP is necessary to 
transcribe SigF, which regulates genes initiating sporulation and therefore is an output of the model. The interplay with other transcription factors AbrB, Hpr, SigA, SigF, SigH and SinR is graphically represented in [5, Figure 3]; SinI inactivates SinR by binding to it. SigA and Signal are considered as an input to the model and are always on. Table 4 lists the Boolean equations building the model in [16] (communicated by the author). They exhibit a certain degree of nondeterminism, since the functions for the off fluents sometimes are not the negation of the on functions. This accounts for incomplete or inconsistent knowledge. In the case of state transitions determined by $k$ conflicting function pairs, we generated $2^{k}$ output states.

Table 4. Boolean rules for the nutritional stress response regulatory network, derived in [16] from [5]. $\bar{x} \hat{=} \neg x, x+y \hat{=} x \vee y, x y \hat{=} x \wedge y$.

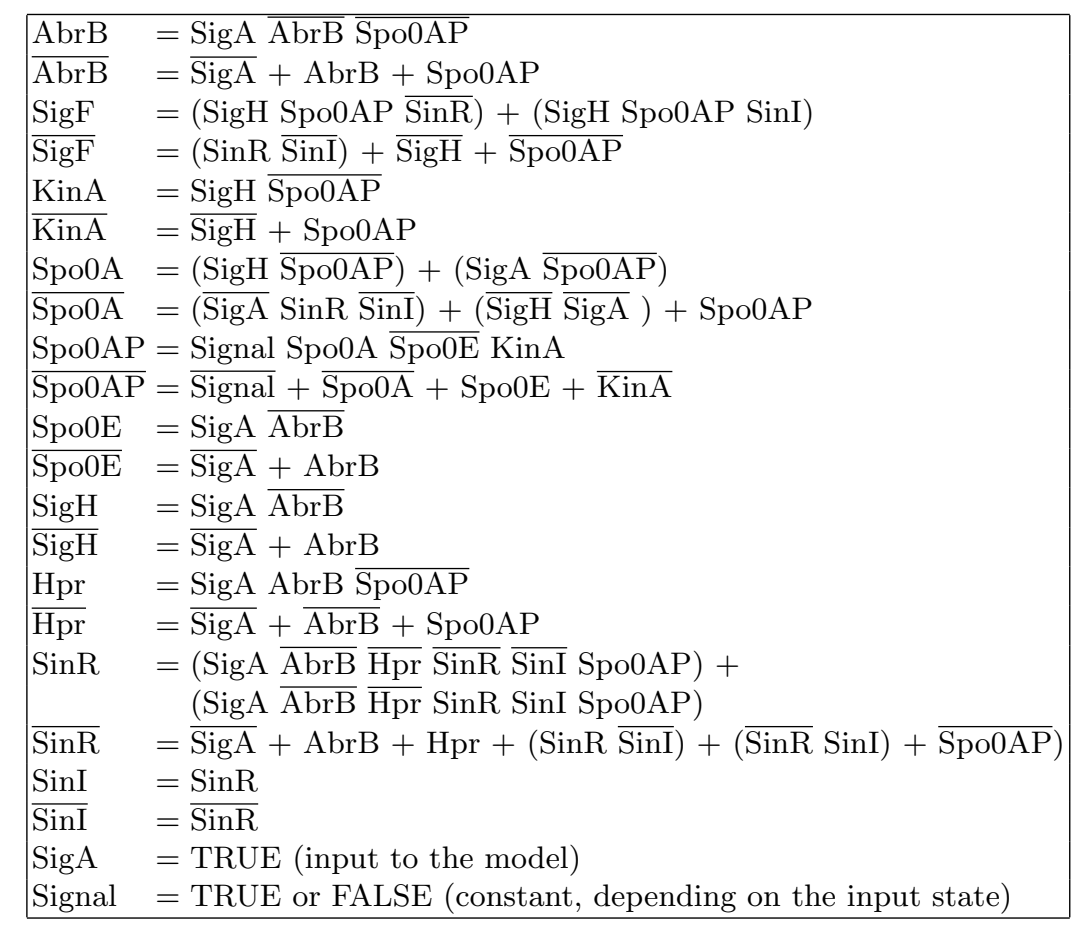

\subsection{Simulation Starting from a State Typical for the Vegetative Growth Phase}

We performed supplementary analyses of the transitions starting from a typical state without the starvation signal [16, Table 4]. The concept lattice for the resulting transitive context (Table 2, with a part of the attribute set only) is shown in Figure 1. The larger circles at the bottom represent object concepts; 
their extents are the four single transitions with the input state at $t=0$ or $t=2$, and the intents are all attributes above a concept. Thus for instance, the two latter transitions have the attribute KinA.in.on in common, designating the respective concept. Moreover, they are distinguished unambigously from other sets of transitions by this attribute - the concept is generated by "KinA.in.on".

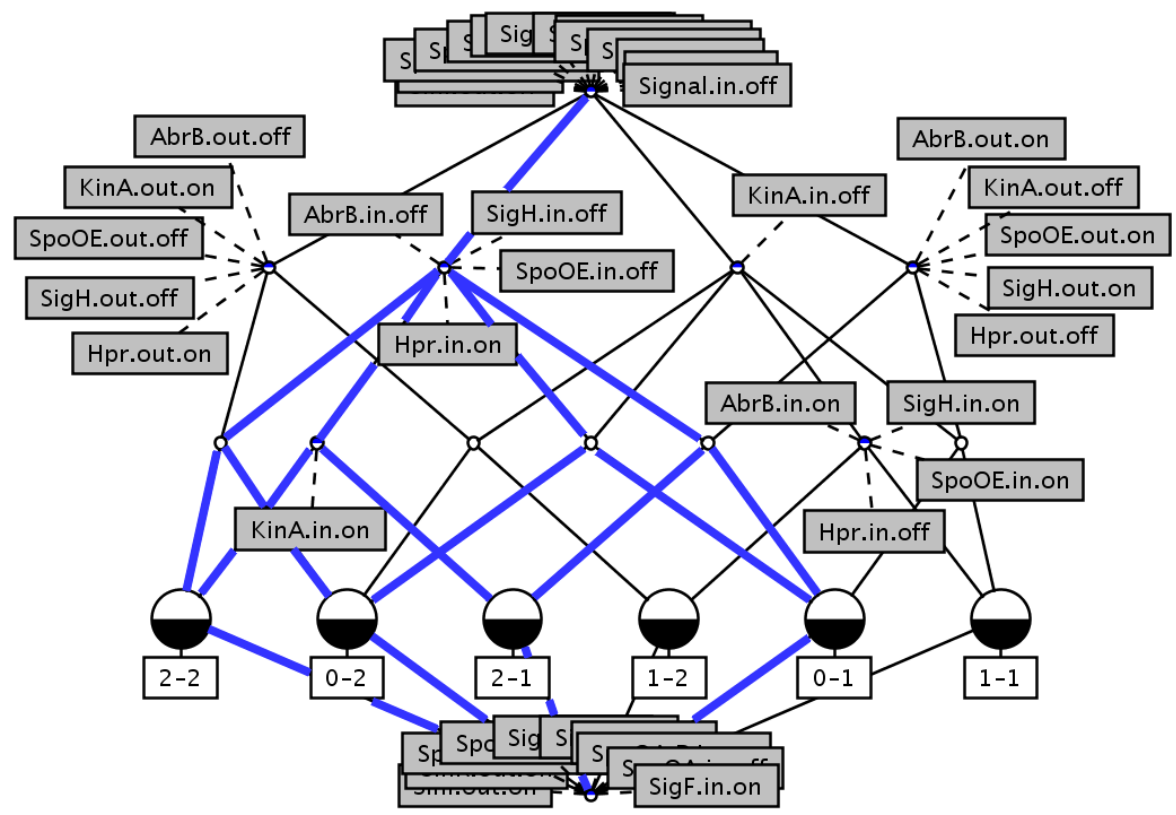

Fig. 1. Concept lattice (computed and drawn with Concept Explorer) representing a simulation without nutritional stress. Signal: starvation; AbrB, Hpr, SigA, SigF, SigH, SinR, Spo0A (phosporylated form SpoOAP): transcription factors; KinA: kinase; Spo0E: phosphatase; SinI inactivates $\operatorname{Sin} R$ by binding to it. $i$ - $j$ indicates a transition $\left(\varphi_{i}^{\text {in }}, \varphi_{j}^{\text {out }}\right)$. Bold / blue lines: Filter (superconcepts) and ideal (subconcepts) of the concept $\left(\left\{\left(\varphi_{0}^{\text {in }}, \varphi_{1}^{\text {out }}\right),\left(\varphi_{0}^{\text {in }}, \varphi_{2}^{\text {out }}\right),\left(\varphi_{2}^{\text {in }}, \varphi_{1}^{\text {out }}\right),\left(\varphi_{2}^{\text {in }}, \varphi_{2}^{\text {out }}\right)\right\}\right.$, \{AbrB.in.off, SigH.in.off, SpoOE.in.off, Hpr.in.on $\})$

Implications of the stem base can be read from the lattice. For instance there are implications between the generators of a concept:

$$
<4>\text { AbrB.in.off } \rightarrow \text { SigH.in.off, SpoOE.in.off, Hpr.in.on }
$$

Analogous implications hold for the attributes of the conclusion, and there are implications between attributes of sub- and superconcepts. $<4>$ indicates that the rule is supported by four transitions.

The bottom concept has an empty extent. Its intent is the set of attributes never occuring during this small simulation. The top concept does not have an 
empty intent - as it is often the case -, but it consists of 10 attributes common to all 6 transitions. The corresponding rule has an empty body $(T)$ :

$$
\begin{aligned}
<6>\top \rightarrow & \text { Signal.in.off, SigA.in.on, SigF.in.off, Spo0A.in.on, Spo0AP.in.off, } \\
& \text { SinR.in.off, SinI.in.off, Signal.out.off, SigA.out.on, SigF.out.off, } \\
& \text { Spo0A.out.on, Spo0AP.out.off SinR.out.off SinI.out.off }
\end{aligned}
$$

Related to the simulation in the presence of nutritional stress, the transitive context has about 20 transitions, 500 concepts and 50 implications. In a such case it is more convenient to query the implicational knowledge base. But also for the visualization of large concept hierarchies, there exist more sophisticated tools like the ToscanaJ suite [http://sourceforge.net/projects/toscanaj/].

\subsection{Analysis of All Possible Transitions}

In order to analyse the dynamics of the B. subtilis network exhaustively, we generated 4224 transitions from all possible $2^{12}=4096$ initial states (thus the rules are nearly deterministic). There were 11.700 transitions in the transitive context, from which we computed the stem base containing 524 implications with support $>0$, but $11.023 .494 \approx 2^{24}$ concepts.

It was not feasible to provide biological evidence for a larger part of the implications, within the scope of this methodological study. This could be done by literature search, especially automatic text mining, by new specialized experiments, or - in a faster, but less reliable way - by comparison with high-throughput observed time series $[18,3.2]$. Instead we will give examples for classes of implications that can be validated or falsified during attribute exploration in specific ways. We start with the examples of $[16,4.3]$.

- "For example, we know that in the absence of nutritional stress, sporulation should never be initiated [5]. We can use model checking to show this holds in our model by proving that no reachable state exists with $\mathrm{SigF}=1$ starting from any initial state in which Signal $=0, \operatorname{SigF}=0$ and Spo0AP $=0 . "[16$, 341] This is equivalent to the rule following from the stem base:

$$
\text { Signal.in.off, SigF.in.off, Spo0AP.in.off } \rightarrow \text { SigF.out.off, }
$$

- SigF.out.on $\rightarrow$ KinA.out.off, Spo0A.out.off, Hpr.out.off, AbrB.out.off: Spo0AP is reported to activate the production of SigF but also repress its own production (mutual exclusion). [5]

- SigH.out.off $\rightarrow$ AbrB.out.off, SpoOE.out.off, SinR.out.off, SinI.out.off All these genes are regulated $\overline{\text { gene.out }}=\overline{\operatorname{SigA} . i n}+\operatorname{AbrB.in}(+\ldots)$.

In our approach, such dependencies and mutual exclusions can be checked systematically. We searched the stem base for further interesting and simple 
implications:

$$
\begin{aligned}
& <4500>\text { Spo0AP.in.on, KinA.out.off } \rightarrow \text { Hpr.out.off } \\
& <4212>\text { SigH.in.on. KinA.out.off } \rightarrow \text { Hpr.out.off } \\
& <3972>\text { AbrB.in.off, KinA.out.off } \rightarrow \text { Hpr.out.off }
\end{aligned}
$$

$\overline{\mathrm{Hpr}}$ and $\overline{\mathrm{KinA}}$ are determined by different Boolean functions, but they are coregulated in all states emerging from any input state characterized by the single attributes Spo0AP.on, SigH.on or AbrB.on.

$$
\begin{aligned}
<3904>\text { AbrB.out.on } \rightarrow & \text { SigA.in.on, SigA.out.on, SigF.out.off, } \\
& \text { Spo0A.out.on, Spo0E.out.on, SigH.out.on, } \\
& \text { Hpr.out.off, SinR.out.off, SinI.out.off }
\end{aligned}
$$

AbrB is an important "marker" for the regulation of many genes, which is understandable from the Boolean rules with hindsight. By a PubMed query, a confirmation was found for downregulation of SigF together with upregulation of $\mathrm{AbrB}$ [17].

Finally we entered sets of interesting attributes as facts into the PROLOG knowledge base, such that a derived implication was computed ${ }^{6}$. Complementary to (9), we searched after conditions for the switch towards sporulation (SigF.out.on) and found the implication

SigF.in.off, Spo0AP.in.off, SigF.out.on

$\rightarrow$ Signal.in.on. Signal.out.on, SigA.in.on, SigA.out.on, Spo0AP.out.off,

Spo0A.out.off, AbrB.out.off, KinA.out.off, Hpr.out.off.

The latter four attributes follow immediately from the Boolean rules, but Spo0AP.out.off depends in a more complex manner on the premises. It is also noteworthy that the class of input states developing to a state with attribute SigF.out.on is only characterized by the common attributes Signal.in.on and SigA.in.on, i.e. the initial presence or absence of no other gene is necessary for the initiation of sporulation ${ }^{7}$

\section{Discussion}

The present work translates observations and simulations of discrete temporal transitions into the language of formal concept analysis. The application to a well studied gene regulatory network showed how a model can be validated in a systematic way, by drawing clear and complete consequences from the theory (the knowledge based network), and we found interesting new transition rules.

\footnotetext{
${ }^{6}$ and accordingly the closure of the attribute set

${ }^{7}$ For this complete simulation, the conditions Signal $=$ SigA $=$ TRUE had been dropped, but they were supposed to be constant.
} 
The approach could be expanded by accounting for the change of the network structure itself in strongly different biological situations, e.g. with or without stress. Thus in ongoing work we adapt a literature based network to observed transcriptome time series, resulting in two sets of Boolean functions related to the stimulation of human fibroblast cells by the cytokines $\operatorname{Tnf} \alpha$ or $\operatorname{Tgf} \beta$.

Until now we have established the foundation in order to exploit manyfold mathematical results of FCA for the analysis of gene expression dynamics and of discrete temporal transitions in general. An important question is: How can attribute exploration be split into partial problems, in this special case? For instance, one could focus on a specific set of genes first, which is understandable as a scaling [4, Definition 28]. Then the decomposition theory of concept lattices will be useful, which permits an elegant description by means of the corresponding formal contexts. [4, Chapter 4]

The price of the logical completeness is its computational complexity. In this regard the status of attribute exploration has not yet fully been clarified. Computation time strongly depends on the logical structure of the context, and there exist cases where the size of the stem base is exponential in the size of the input [10]. However, deriving an implication from the stem base is possible in linear time, related to the size of the base, and the PROLOG queries in Section 3.2 were very fast. As demonstrated in Section 2.4, attribute exploration can be shortened by background knowledge. Further it will be crucial to decide implications without the necessity to generate all possible transitions. For that purpose, model checking [6] could be a promising approach, or the structural and functional analysis of Boolean networks by an adaptation of metabolic network methods in [9]. There, determining activators or inhibitors corresponds to the kind of rules found by our method, and logical steady state analysis indicates which species can be produced from the input set and which not. An exciting direction of research would be to conclude dynamical properties of Boolean networks from their structure and the transition functions, e.g. by regarding them as polynomial dynamical systems over finite fields [12, Section 4] and by exploiting theoretical work in the context of cellular automata [12, Section 6].

The present work is a first step to use the potential of formal concept analysis for solving questions within systems biology. As indicated, many directions of research are possible. We encourage their investigation and are open to any collaboration with mathematicians, computer scientists or (systems) biologists.

Acknowledgement. The work was supported by the German Federal Ministry of Education and Research BMBF (FKZ 0313652A).

\section{References}

1. Chabrier-Rivier, N. et al.: Modeling and Querying Biomolecular Interaction Networks. Theor. Comp. Sc. 325(1), 25-44 (2004)

2. Choi, V., et al.: Using Formal Concept Analysis for Microarray Data Comparison. Advances in Bioinformatics and Computational Biology 5, 57-66 (2006) 
3. Ganter, B., Stumme, S., Wille, R.: Formal Concept Analysis - Foundations and Applications. LNAI, vol. 3626. Springer, Heidelberg (2005)

4. Ganter, B., Wille, R.: Formal Concept Analysis - Mathematical Foundations. Springer, Heidelberg (1999)

5. de Jong, H., et al.: Qualitative Simulation of the Initiation of Sporulation in Bacillus subtilis. Bulletin of Mathematical Biology 66, 261-299 (2004)

6. Esparza, J.: Model checking using net unfoldings. Sci. Comput. Programm. 23, 151195. (1994)

7. von Hentig, H.: Magier oder Magister? Über die Einheit der Wissenschaft im Verständigungsprozess. Suhrkamp, Frankfurt (1974)

8. Kauffman, S.A.: The Origins of Order: Self-Organization and Selection in Evolution. Oxford University Press, New York (1993)

9. Klamt, S., et al.: A methodology for the structural and functional analysis of signaling and regulatory networks. BMC Bioinformatics 7(56) (2006)

10. Kuznetsov, S.O., Obiedkov, S.A.: Counting Pseudo-intents and \#P-completeness. In: Missaoui, R., Schmid, J. (eds.) ICFCA 2006. LNCS, vol. 3874, pp. 306-308. Springer, Heidelberg (2006)

11. King, R.D., Garrett, S.W, Coghill, G.M.: On the Use of Qualitative Reasoning to Simulate and Identify Metabolic Pathways. Bioinformatics 21(9), 2017-2026 (2005)

12. Laubenbacher, R.: Algebraic Models in Systems Biology. In: Anai, H., Horimoto, K. (eds.) Algebraic Biology 2005, pp. 33-35. Universal Academy Press, Tokyo (2005)

13. Motameny, S., Versmold, B., Schmutzler, R.: Formal Concept Analysis for the Identification of Combinatorial Biomarkers in Breast Cancer. In: Medina, R., Obiedkov, S.A. (eds.) ICFCA 2008. LNCS, vol. 4933, pp. 229-240. Springer, Heidelberg (2008)

14. Peirce, C.S.: How to Make Our Ideas Clear. In: Hartshorne, C., Weiss, P. (eds.) Collected papers. Harvard University Press, Cambridge / Mass. (1931-35)

15. Ganter, B., Rudolph, S.: Formal Concept Analysis Methods for Dynamic Conceptual Graphs. In: ICCS 2001, LNAI 2120, pp. 143-156. Springer, Heidelberg (2001)

16. Steggles, L. J. et al.: Qualitatively modelling and analysing genetic regulatory networks: a Petri net approach. Bioinformatics 23(3), 336-343 (2007)

17. Tomas, C.A. et al.: DNA array-based transcriptional analysis of asporogenous, nonsolventogenic Clostridium acetobutylicum strains SKO1 and M5. J Bacteriol. 15, 4539-47 (2003)

18. Wollbold, J.: Attribute Exploration of Discrete Temporal Transitions. In: Gély, A., et al. (eds.) Contributions to ICFCA 200\%, pp. 121-130. Clermont-Ferrand (2007)

19. Wollbold, J., Huber, R., Wolff, K.E.: Conceptual Representation of Gene Expression Processes. In: Knowledge Processing in Practice 200\%. To appear in LNAI. Springer, Heidelberg (2008)

20. Zickwolff, M.: Rule Exploration: First Order Logic in Formal Concept Analysis. PhD thesis. University of Technology, Darmstadt (1991) 